\title{
Respostas cardiorespiratórias em exercícios de hidroginástica executados com e sem o uso de equipamento resistivo
}

\author{
Stephanie S. Pinto \\ Cristine L. Alberton \\ Márcio E. Becker \\ Mabel M. Olkoski \\ Luiz F.M. Kruel
}

\author{
U niversidade Federal do Rio Grande do Sul \\ UFRGS \\ Porto A legre \\ Rio Grande do Sul \\ Brasil
}

\section{RESUMO}

O objectivo do presente estudo foi analisar a frequência cardíaca e o consumo de oxigénio em exercícios de hidroginástica executados com e sem equipamento resistivo. A amostra foi composta por dez mulheres jovens que realizaram duas sessões de testes aquáticos, com os exercícios jumping jack e cross country sky realizados nas situações sem equipamento resistivo, com equipamento aquafins e com equipamento aqualogger, respeitando intervalos de 30 minutos. Para a coleta de dados de frequência cardíaca foi utilizado um frequencímetro $S 610$ (POLAR) e para o consumo de oxigênio, um analisador de gases KB1-C (AEROSPORT). Utilizou-se ANOVA para medidas repetidas, post-hoc de Bonferroni e teste-t pareado $(p<0.05)$. O cross country sky comparado ao jumping jack apresentou um comportamento mais elevado para ambas as variáveis, provavelmente, devido a uma maior amplitude de movimento e massa muscular envolvida no exercício. Ao compararmos as diferentes situações, no cross country sky foi observado um aumento estatisticamente significativo do consumo de oxigénio da situação sem equipamento para as situações com equipamento, enquanto que no jumping jack não foram observadas diferenças entre as situações. Já a frequência cardíaca apresentou diferenças estatisticamente significativas entre as três situações para ambos os exercícios. Assim, o uso do equipamento resistivo pode ser eficaz para aumentar as respostas cardiorespiratórias associado ao exercício realizado.

Palavras-chave: consumo de oxigénio, frequência cardíaca, hidroginástica, equipamento resistivo.

\section{ABSTRACT \\ Cardiorespiratory responses induced by hydrogymanstics ex ercis- es performed with and without the use of resistive equipment}

The aim of the present study was to analyse the heart rate and the oxygen uptake in hydrogymnastics exercises performed with and without the use of resistive equipment. The sample has been composed by ten young women who have undergone two sessions of aquatic tests, with the exercises jumping jack and cross country sky performed in the situations without resistive equipment, with the use of the aquafins equipment and with the use of the aqualogger equipment, which respected 30 -minute breaks. For collecting heart rate and oxygen uptake data a S610 frequencimeter (POLAR) and a KB1-C gas analyser ( $A E R O S P O R T$ ) were respectively used. ANOVA was used for repetitive measures, post-hoc Bonferroni and paired t-test $(p<0.05)$. The cross country sky compared to the jumping jack showed a higher behavior for both variables due, probably, to a greater movement amplitude and greater muscle mass involved during the execution of the exercise. A $n$ increase statistically significant in the oxygen uptake has been observed in the cross country sky from the situation without equipment when compared with the situations with equipment. $0 \mathrm{n}$ the other hand, in the jumping jack have been noticed no differences between the situations. However, the heart rate has presented differences statistically significant among the three situations for both exercises. Therefore, the use of resistive equipment may be efficient in order to increase the cardiorespiratory responses related to the exercise done.

Key-words: oxygen consumption, heart rate, hydrogymnastics, resistive equipment. 


\section{INTRODUÇÃO}

Exercícios aquáticos na posição vertical, tal como a hidroginástica, estão sendo cada vez mais indicados devido aos seus diversos benefícios a saúde $(9,11)$. Essa modal idade pode promover melhoria nos diversos componentes da aptidão física, como força $(4,6,15$, $20,23)$, flexibilidade (2, 23), composição corporal (23) e condicionamento cardiorespiratório (2, 3, 23, 24). Além disso, exercícios realizados no meio aquático apresentam um reduzido impacto comparado com o meio terrestre $(11,14)$ e um comportamento de frequência cardíaca e pressão arterial mais baixos (17, 22). Os benefícios que o meio aquático proporciona no condicionamento geral do corpo estão relacionados com as propriedades físicas da água. Podemos destacar, dentre essas propriedades, a resistência ao avanço (R), que pode ser expressa como $R=0,5 \rho$ $A v^{2} C d$, onde, $\rho$ é a densidade do fluido, $A$ é a área de superfície projetada, $v$ é a velocidade do movimento e C d é o coeficiente de arrasto (18).

Na hidroginástica podemos utilizar exercícios que produzam uma maior resistência na água, através do aumento da velocidade de execução ou através da alteração da área projetada, que pode ser modificada pela utilização do corpo de diferentes formas na água e também pela execução de exercícios com uso de implementos resistivos $(1,7,10)$. O aumento da resistência através do aumento da velocidade do movimento, entretanto, só é produzido enquanto esse mantiver a sua total amplitude. A partir de determinada velocidade de execução, o movimento, ao ser acelerado, é encurtado, e assim o aumento da velocidade é compensado pela diminuição da área projetada. Estudos envolvendo a utilização de equipamentos resistivos em um treinamento de força na hidroginástica foram realizados com o objectivo de analisar a influência de seu uso no incremento da força muscular, detendo respostas positivas $(4,6)$. Outros estudos foram realizados com a análise eletromiográfica e demonstraram que a sua utilização pode aumentar a atividade muscular de determinadas musculaturas para cadências específicas $(5,19)$. Porém, na literatura pesquisada, não foram encontrados estudos que relacionassem os exercícios de hidroginástica executados com e sem o uso de equi pamento resistivo e suas respectivas respostas cardiorespiratórias. Portanto, o objectivo deste estudo foi analisar as respostas da frequência cardíaca (FC) e do consumo de oxigénio
$\left(\mathrm{VO}_{2}\right)$ em exercícios de hidroginástica com e sem o uso de equipamento resistivo.

\section{METODOLOGIA}

A amostra deste estudo foi composta por 10 mulheres jovens ambientadas ao meio aquático, selecionadas por voluntariedade, com idades entre 20 e 23 anos. Todas eram isentas de problemas físicos e medicação e assinaram um termo de consentimento informado, no qual contavam todas as informações pertinentes ao estudo. Suas características são apresentadas na Tabela 1 . Os critérios estabelecidos para a coleta dos dados foram: não alimentar-se no período de 3 a 4 h antes do início da sessão de testes, sem a ingesta de estimulantes e evitar a prática de atividades físicas intensas durante as últimas $24 \mathrm{~h}$ (8).

Tabela 1. Médias e desvios padrão ( $X \pm D P$ ) das variáveis de caracterização da amostra "idade", "massa", "estatura" e "VO ${ }_{2 \text { pico }}$.

\begin{tabular}{ccccc}
\hline $\begin{array}{c}\text { Idade } \\
\text { (anos) }\end{array}$ & Massa $(\mathrm{kg})$ & $\begin{array}{c}\text { Estatura } \\
(\mathrm{cm})\end{array}$ & $\begin{array}{c}\mathrm{VO}_{{ }_{\text {2pico }}}(\mathrm{ml} . \mathrm{kg} \\
{ }^{1} \cdot \mathrm{min}^{-1} \text { ] }\end{array}$ \\
\hline$X \pm D P$ & $21.9 \pm 0.99$ & $56.91 \pm 5.47$ & $160.7 \pm 5.96$ & $40.24 \pm 5.42$ \\
\hline \multicolumn{4}{c}{ Nota: $\mathrm{VO}_{\text {2pico }}-$ consumo de oxigénio de pico. }
\end{tabular}

Na primeira sessão de avaliação, foram realizadas as medidas da massa corporal e da estatura, em uma balança de al avanca FI LIZO LA , com estadiômetro acoplado, e um teste de $\mathrm{VO}_{2}$ máximo, em uma esteira 10200 ATL, da marca IN BRAMED (Porto Alegre, Brasil). A seguir foram realizadas duas sessões de testes aquáticos no Centro Natatório da Escola de Educação Física da Universidade Federal do Rio Grande do Sul. Em cada sessão os indivíduos realizaram um dos dois exercícios no meio aquático, o jumping jack (J) ou o cross country sky (CCS) (Figura 1), que tiveram suas ordens randomizadas e foram executados com um intervalo mínimo de 48h.

Figura 1. Fase inicial e final dos exercícios realizados no meio aquático.
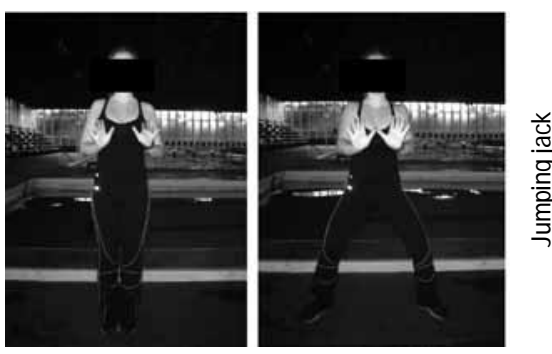

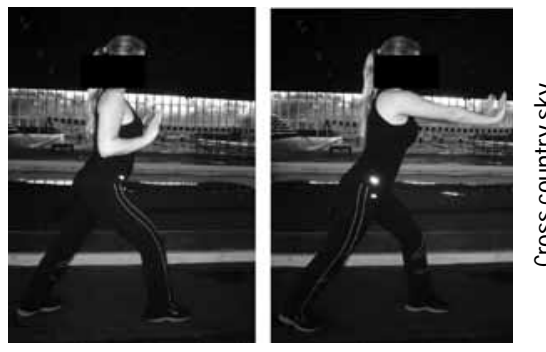

No início de cada sessão de testes, o indivíduo permanecia inicialmente 10 minutos em repouso em decúbito dorsal e 5 minutos em repouso na posição ortostática, para avaliação do $\mathrm{VO}_{2}$ e da $\mathrm{FC}$ de repouso. A pós iniciava-se o procedimento em exercício. Cada exercício foi realizado na cadência de 80 bpm, que foi reproduzida por um metrônomo modelo MATRIX MR-500 da marca QUARTZ, durante 4 minutos, nas situações sem uso de equipamento resistivo (SEM), com uso do equipamento aquafins (FINS) e com uso do equipamento aqualogger (LOGGER), que foram randomizadas e respeitaram intervalos de 30 minutos.

Para verificação da FC foi utilizado um cardio-freqüêncimetro 5610 , da marca POLA $R$, já para a verificação do $\mathrm{VO}_{2}$ foi utilizado um analisador de gases portátil KB1-C, da marca AEROSPORT (Ann Arbor, USA). Os valores de ambas as variáveis foram obtidos a cada 20 segundos. Para análise desses dados, utilizou-se a média dos valores coletados entre o terceiro e o quarto minuto de cada exercício em cada situação, correspondente a $\mathrm{FC}$ e ao $\mathrm{VO}_{2}$ em steady state (12). Os exercícios de hidroginástica foram realizados em uma piscina com profundidade entre 1.10 e $1.40 \mathrm{~m}$, permitindo que a profundidade de imersão ficasse entre o processo xifóide e ombros. A temperatura da água foi controlada e mantida entre 32 e $33^{\circ} \mathrm{C}$, para evitar a sua influência nas respostas fisiológicas (16). Foram tomados al guns cuidados para a local ização dos equipamentos resistivos nos indivíduos. $O$ equipamento aqualogger era posicionado imediatamente acima do maléolo medial, com o velcro para frente. $\mathrm{O}$ equipamento aquafins, no exercício JJ era posicionado $3 \mathrm{~cm}$ acima do maléolo lateral e no exercício CCS, $3 \mathrm{~cm}$ acima do maléolo medial, com as abas colocadas por forma a criar resistência aos movimentos contra-laterais (JJ) ou ântero-posteriores (CCS).
Para a análise dos dados coletados foi utilizada estatística descritiva, com valores de média \pm desvio padrão $(X \pm D P)$. Verificamos a normalidade dos dados através do teste de Shapiro-Wilk $(p>0.05)$ e os resultados obtidos justificam a utilização da estatística paramétrica para as análises subsequentes. Para a análise da $\mathrm{FC}$ e do $\mathrm{VO}_{2}$ de repouso entre os dois dias de avaliação eentre os dois exercícios na mesma situação, foi utilizado o Teste-t pareado. Já para a análise da $\mathrm{FC}$ e do $\mathrm{VO}_{2}$ entre as diferentes situações foi utilizado ANOVA para medidas repetidas, com o teste post-hoc de Bonferroni. O nível de significância adotado neste estudo foi $p<0.05$. Todos os testes estatísticos foram processados no programa estatístico SPSS versão 11.0.

\section{RESULTADOS}

Os resultados referentes à $\mathrm{FC}$ e ao $\mathrm{VO}_{2}$ de repouso não apresentaram diferenças estatisticamente significativas entre os dois dias de avaliação, tanto na posição de decúbito dorsal, como na posição ortostática, conforme a Tabela 2. Esses resultados demonstram que os indivíduos iniciaram os testes nos dois dias com a FC e o $\mathrm{VO}_{2}$ de repouso semelhantes, indicando que a magnitude das alterações encontradas nessas variáveis durante o exercício pode ser atribuída ao esforço para execução do mesmo.

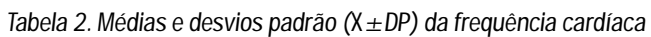
e do consumo de oxigénio de repouso entre os dois dias de avaliação nas posições corporais decúbito dorsal e ortostática.

\begin{tabular}{cccccc}
\hline & \multicolumn{3}{c}{$\mathrm{VO}_{2}\left(\mathrm{ml}^{\circ} \mathrm{kg}^{-1} \cdot \mathrm{min}^{-1}\right)$} & \multicolumn{2}{c}{$\mathrm{FC}(\mathrm{bpm})$} \\
\hline Posição & Coleta & $X \pm \mathrm{DP}$ & $\mathrm{Sig}$. & $X \pm \mathrm{DP}$ & Sig. \\
& & & & & \\
\hline DD & $1^{\circ}$ dia & $3.27 \pm 0.47$ & 0.196 & $64.46 \pm 5.97$ & 0.612 \\
& $2^{\circ}$ dia & $2.88 \pm 0.82$ & & $65.6 \pm 3.39$ & \\
\hline OR & $1^{\circ}$ dia & $3.74 \pm 0.34$ & 0.364 & $87.38 \pm 13.79$ & 0.725 \\
& $2^{\circ}$ dia & $3.52 \pm 0.73$ & & $85.57 \pm 10.49$ & \\
\hline
\end{tabular}

Nota: $\mathrm{FC}$ - frequência cardica; $\mathrm{VO}_{2}$ - consumo de oxigénio; $\mathrm{DD}$ - decúbito dorsal; $\mathrm{OR}$ - ortostática. $\mathrm{p}<0.05$.

Os resultados da $\mathrm{FC}$ e do $\mathrm{VO}_{2}$ para cada exercício entre as diferentes situações são apresentados na Tabela 3. Ao compararmos as diferentes situações, no exercício CCS foi observado um aumento significativo do $\mathrm{VO}_{2}$ da situação SEM para as situações LOGGER e FINS, sem diferenças estatisticamente 
significativas entre os equipamentos. Já a FC apresentou diferença estatisticamente significativa entre as três situações. $\mathrm{O}$ exercício JJ não apresentou diferenças estatisticamente significativas para o $\mathrm{VO}_{2}$ entre as situações, enquanto que para a FC foram observadas diferenças estatisticamente significativas entre elas.

Tabela 3. Médias e desvios padrão (X土 DP) da frequência cardíaca e do consumo de oxigénio durante os exercícios cross country sky e jumping jack nas situações sem equipamento resistivo, com equipamento aqualogger e com equipamento aquafins.

\begin{tabular}{|c|c|c|c|c|c|c|}
\hline & \multicolumn{3}{|c|}{ Cross country sky } & \multicolumn{3}{|c|}{ Jumping Jack } \\
\hline & SEM & LOGGER & FINS & SEM & LOGGER & FINS \\
\hline $\mathrm{VO}_{2}$ & $17.87 \pm 4.03^{\mathrm{a}}$ & $22.13 \pm 4.62^{b}$ & $22.90 \pm 5.26^{\mathrm{b}}$ & $12.47 \pm 2.16^{\mathrm{a}}$ & $13.75 \pm 1.99^{\mathrm{a}}$ & $15.13 \pm 2.28^{\mathrm{a}}$ \\
\hline $\mathrm{FC}$ & $130.58 \pm 16.41^{a}$ & $145.27 \pm 20.71^{b}$ & $154.33 \pm 18.95^{c}$ & $108.50 \pm 4.48^{a}$ & $117.07 \pm 5.61^{b}$ & $122.40 \pm 9.06^{\circ}$ \\
\hline
\end{tabular}

Nota: $\mathrm{FC}$ - frequência cardíaca; $\mathrm{VO}_{2}$ - consumo de oxigénio; SEM - situação sem equipamento resistivo; LOGGER situação com equipamento aqualogger; FINS - situação com equipamento aquafins. * letras diferentes representam diferenças significativas $(p<0.05)$ entre as situações.

Os resultados da $\mathrm{FC}$ e do $\mathrm{VO}_{2}$ entre os dois exercícios executados em cada uma das situações são apresentados nas Figuras 2 e 3, respectivamente. Ao compararmos os dois exercícios, todas as situações apresentaram um comportamento significativamente mais elevado da $\mathrm{FC}$ e do $\mathrm{VO}_{2}$ para o CCS comparado ao JJ.

Figura 2. Frequência Cardíaca (FC) em cada situação entre os exercícios cross country sky (CCS) e jumping jack (J J).

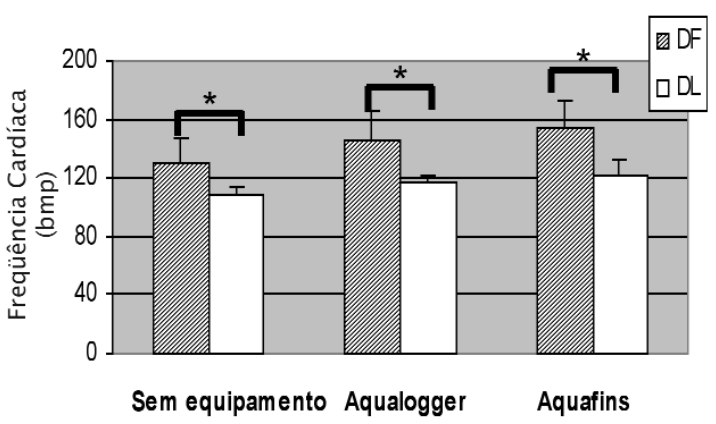

* representa diferenças estatisticamente significativas para um $p<0.05$

Figura 3. Consumo de oxigénio $\left(\mathrm{VO}_{2}\right)$ em cada situação entre os exercícios cross country sky (CCS) e jumping jack (J J).

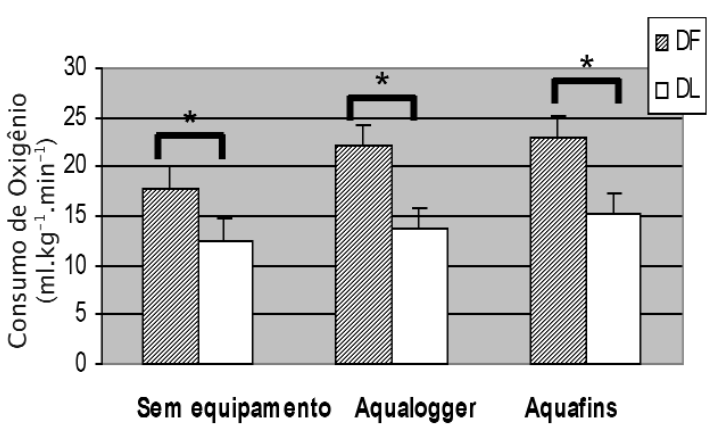

* representa diferenças estatisticamente significativas para um $p<0.05$

\section{DISCUSSÃO}

As respostas cardiorrespiratórias nos exercícios de hidroginástica parecem ser modificadas tanto pela escolha do exercício, como pela sua respectiva velocidade de execução (1). Os efeitos agudos dos exercícios realizados no meio aquático são influenciados pelas propriedades físicas da água. Portanto, podemos utilizar exerćcios que produzam uma mai or resistência na água ( $\left.R=0,5 \rho A V^{2} C d\right)$, em função da maior densidade do meio líquido $(\rho)$ comparada a densidade do ar, pelo aumento da área projetada $(A)$ e/ ou aumento da velocidade de execução ( $\left.\mathrm{V}^{2}\right)$ (18). Conforme os resultados do presente estudo, observamos que é possível ocorrer um aumento da FC e do $\mathrm{VO}_{2}$ com a utilização de equi pamentos resistivos durante a execução de determinados exercícios de hidroginástica em ritmos de execução iguais. Esses dados corroboram com outros estudos que também encontraram um aumento nas respostas de $\mathrm{FC} \mathrm{e} \mathrm{VO}{ }_{2}$ conforme $o$ aumento da intensidade de execução dos exercícios (1, 7, 13, 21). No caso do estudo de Alberton et al. (1), essa intensidade foi alterada pela escolha de diferentes exercícios modificando a área projetada, e também pela exe cução desses em diferentes cadências, alterando a velocidade de execução. Em nosso estudo, essa intensidade, de acordo com os resultados obtidos, foi modificada pela utilização ou não de implemento resistivo e pela escol ha do exercício, explorando a área de diferentes formas e consequentemente a resistência da água. 
A influência da escolha do exercício sobre as respostas cardiorespiratórias pode ser explicada também pela diferença da resistência ao avanço provocada pela execução do mesmo. Como podemos ver nos resultados, só houve um aumento significativo nas respostas de $\mathrm{VO}_{2}$ durante a execução do exercício cross country sky da situação sem equipamento resistivo para as situações com uso de equipamento resistivo. Já no exercício jumping jack não foi encontrado um aumento significativo nas respostas de $\mathrm{VO}_{2}$ para as mesmas situações. No nosso estudo, esse resultado provavelmente pode ser explicado pelo fato do exercício cross country sky apresentar uma maior amplitude de movimento do que o exercício jumping jack, sendo que ambos foram executados em uma mesma unidade de tempo. Além disso, há uma mai or massa muscular envolvida na execução do mesmo.

Alguns estudos analisaram a influência do uso de equipamento resistivo em determinadas variáveis. Dentre esses podemos citar os que analisaram os efeitos de um programa de treinamento de força com e sem o uso de equipamento resistivo (4, 6). Nesses estudos foi encontrado um incremento da força muscular, de determinados grupos musculares, independente da utilização ou não de diferentes equipamentos resistivos. Esses resultados nos mostram que provavelmente o uso de equipamento resistivo faz com que os indi víduos executem o exercício em uma velocidade máxima menor que a velocidade máxima obtida sem equi pamento, entretanto ambas as situações parecem produzir uma resistência na água similar (4). Já o estudo de Black (5) teve como objectivo analisar a atividade elétrica dos músculos reto femoral e bíceps femoral durante o exercício de flexão e extensão do quadril no meio líquido com e sem a utilização do equipamento resistivo aquafins, em três cadências (40,60, 80 bpm) e na velocidade máxima de execução. Os exercícios, quando realizados nas cadências de 40 e 80 bpm, apresentaram um aumento significativo da ativação muscular do reto femoral com a utilização do equipamento resistivo. Contudo, os resultados nos mostram que a situação que apresenta a maior atividade elétrica muscular é a máxima velocidade, independente do uso ou não do equipamento. Esses últimos resultados corroboram com o estudo de Pöyhönen et al. (19), que analisaram a atividade eletromiográfica da musculatura do quadríceps e dos ísquio-tibiais no exercício de flexão e extensão do joel ho no meio líquido, com e sem o uso de equipamento, e encontraram uma ativação semel hante para ambas as situações na máxima velocidade. Se fizermos uma relação entre os estudos citados acima, os quais não analisaram as respostas cardiorespi ratórias decorrentes da real ização dos exercícios, e o presente estudo, podemos dizer que obtivemos uma resposta positiva ao uso de equipamentos resistivos. Com base em nossos resultados, verificamos que o uso desses implementos pode aumentar a magnitude das respostas de $\mathrm{FC} \mathrm{e} \mathrm{VO}{ }_{2}$. Isso se deve ao fato de termos fixado a velocidade de execução em 80 bpm, tanto para o exercício sem a utilização do equipamento resistivo como para o mesmo com a utilização de diferentes equi pamentos resistivos. Dessa forma, o indivíduo executava o mesmo exercício, na mesma velocidade, aumentando a resistência da água através do aumento da área projetada. De acordo com os resultados da literatura e do presente estudo, parece-nos poder concluir que a escoIha do exercício, a velocidade de execução e o uso de equipamento resistivo, sendo trabal hados em conjunto, podem otimizar as respostas cardiorespiratórias em exercícios de hidroginástica. Cabe ressaltar que, de acordo com a literatura, parece ser fundamental, para modular a intensidade do exercício, a velocidade de execução. Portanto, nas aulas de hidrogi nástica devemos conhecer as limitações de nossos alunos, ou seja, caso esses consigam manter a velocidade de execução proposta para determinado exerć́cio associado ao uso do equipamento resistivo, torna-se eficaz a prática de exercícios de hidroginástica com diferentes implementos resistivos com o intuito de maximizar as melhoras cardiorespiratórias.

\section{CORRESPONDÊNCIA}

\section{Stephanie Santana Pinto}

Grupo de Pesquisa em Atividades Aquáticas e Terrestres - Laboratório de Pesquisa do Exercício Escola de Educação Física

Universidade Federal do Rio Grande do Sul

Rua Felizardo, 750 - Bairro Jardim Botânico

CEP 90690-200 - Porto Alegre/ RS

Telefone: (0XX51) 3316-5820

tetisantana@yahoo.com.br 
REFERÊNCIAS BIBLIOGRÁFICAS

1. Alberton CL, Coertjens M, Figueiredo PAP, Kruel LFM (2005). Behavior of oxygen uptake in water exercises performed at different cadences in and out of water. M ed Sci Sports Exerc 37(5): S103.

2. Alves RV, Mota J, Costa MC, Alves JGB (2004). Physical fitness and elderly health effects of hydrogymnastics. Rev Bras M ed Esporte 10(1): 38-43.

3. Avellini BA, Shapiro Y, Pandolf KB (1983). Cardio-respiratory physical training in water and on Land. Eur J A ppl Physiol 50: 255-263.

4. Barella RE, Muller FG, Severo CR, Cardoso AS, Figueiredo PAP, Brentano MA, Kruel LFM (2004). Efeitos de um treinamento de força aplicado em mulheres praticantes de hidroginástica. Rev Bras Fisiol Exerc 3(1): 136.

5. Black GL (2005). A nálise eletromiográfica em exercícios na água com velocidade e resistência variável. Porto Alegre. Dissertação de Mestrado. Universidade Federal do Rio Grande do Sul.

6. Cardoso AS, Tartaruga LAP, Barella RE, Brentano MA, Kruel LFM (2004). Effects of a deep water training program on women's muscle strenght. FIEP Bulletin 74: 590593.

7. Cassady SL, Nielsen DH (1992). Cardiorespiratory Responses of Healthy Subjects to calisthenics Performed on Land Versus in Water. Phys Ther 75: 532-538.

8. Cooke CB (1996). Metabolic rate and energy balance. In: Eston R, Reilly T. Kinanthropometry and exercise physiology laboratory manual. London: E \& FN Spon, 175-195.

9. Eckerson J, Anderson T (1992). Physiological response to water aerobics. J Sports M ed Phys Fitness 32(3): 255-261.

10. Johnson BL, Stromme SB, Adamczyk JW, Tennoe KO (1977). Comparison of oxygen uptake and heart rate during exercises on land and in water. Phys Ther 57(3): 273-278.

11. Kruel LFM (2000). A lterações fisiológicas e biomecânicas em indivíduos praticando exercícios de hidroginástica dentro e fora d'água. Santa Maria. Tese de Doutorado. Universidade Federal de Santa Maria.

12. Linnarsson D (1974). Dynamics of pulmonary gas exchange and heart rate changes at start and end of exercise. A cta Physiol Scand Suppl 415: 1-68.

13. McArdle WD, Magel JR, Lesmes GR, Pechar GS (1976). Metabolic and cardiovascular adjustment to work in air and water at 18, 25 and 33ㄷ. J A ppl Physiol 40: 85-90.
14. Miyoshi T, Shirota T, Yamamoto S, Nakazawa K, Akai M (2004). Effect of the walking speed to the lower limb joint angular displacements, joint moments and ground reaction forces during walking in water. Disabil Rehabil 26(12): 724732.

15. Müller FG (2002). A treinabilidade da força muscular em idosas praticantes de hidroginástica. Florianópolis. Dissertação de Mestrado. Universidade do Estado de Santa Catarina.

16. Müller FG, Alberton CL, Tartaruga LAP, Kruel LFM (2005). Freqüência Cardíaca em homens imersos em diferentes temperaturas de água. Rev Port Cienc D esp 5(3): 266273.

17. Nakanishi Y, Kimura T, Yoko Y (1999). Maximal responses to deep water running at termoneutral temperature. A ppl Human Sci 18(2): 31-35.

18. Pöyhönen T, Keskinen KL, Hautala A, Mälkiä E (2000). Determination of hydrodinamic drag forces and drag coefficients on human leg/foot model during knee exercise. Clin Biomech 15: 256-260.

19. Pöyhönen $T$, Kyrolainen $H$, Keskinen $K L$, Hautala A, Savolainen J, Mälkiä E (2001). Electromyographic and kinematic analysis of therapeutic knee exercises under water. Clin Biomech 16: 496-504.

20. Pöyhönen T, Sipilä S, Keskinen KL, Hautala A, Savolainen J, Mälkiä E (2002). Effects of aquatic resistance training on neuromuscular performance in healthy women. M ed Sci Sports Exerc 34(12): 2103-2109.

21. Robert JJ, Jones L, Bobo M (1996). The physiologic response of exercising in the water and on land with and without the X1000 walk'n tone exercise belt. Res Q Exerc Sport 67(3): 310-315.

22. Srámek P, Simecková M, Janski L, Savlíková J, Vybíral S (2000). Human Physiological responses to immersion into water of different temperatures. Eur J A ppl Physiol 81: 436-442.

23. Takeshima N, Rogers ME, Watanabe WF, Brechue WF, Okada A, Yamada T, Islam MM, Hayano J (2002). Waterbased exercise improves health-related aspects of fitness in older women. M ed Sci Sports Exerc 33(3): 544-551.

24. Tauton JE, Rhodes EC, Wolski LA, Donelly M, Warren J, Elliot J, McFarlane L, Leslie J, Mitchell J, Lauridsen B (1996). Effect of land-based and water-based fitness programs on the cardiovascular fitness, strenght and flexibility of woman aged 65-75 years. Gerontology 42: 204-210. 